A Fundamental Rule:

\title{
Determining the Importance of Flow prior to Polymer Crystallization
}

Claire Mcllroy ${ }^{1, a)}$

School of Mathematics $\&$ Physics, University of Lincoln, Lincoln, UK, LN6 $7 T S$

(Dated: 31 October 2019)

A continuum-level model for non-isothermal polymer crystallization following a complex flow is presented, along with a fundamental rule that may be employed to determine if the flow will influence the ensuing crystallization dynamics. This rule is based on two dimensionless parameters: the (Rouse) Weissenberg number, and an inverse Deborah number defined by the ratio between the time taken to cool to the melting point versus the stretch relaxation time, which determines the time available for flowenhanced crystallization. Moreover, we show how the time to reach the melting point can be derived semi-analytically and expressed in terms of the processing conditions in the case of pipe flow - ubiquitous in polymer processing. Whilst the full numerical model is required to quantitatively predict induction times and spherulite-size distributions, the proposed fundamental rule may be used practically to ensure, or eliminate, flow-enhanced structures by controlling the processing conditions or material properties. We discuss how flow-enhanced structures may be revealed only after post-processing annealing, and finally examine previous works that have successfully applied the model to extrusion-based three-dimensional (3D) printing.

a)Electronic mail: cmcilroy@lincoln.ac.uk 


\section{INTRODUCTION}

Semi-crystalline polymer melts consist of polymer chains that are able to fold into ordered lamellae upon cooling, which make up larger spherulite structures. During processing, polymer crystallization is initiated via a temperature quench. Brief intervals of flow prior to the quench can dramatically enhance the crystallization process, resulting in a higher nucleation density and therefore shorter induction times and significantly smaller spherulites ${ }^{1,2}$. These smaller spherulite structures improve the material properties relative to quiescent crystallization $^{3}$, thus flow-enhanced crystallization is often considered favorable in polymer processing. However, variations in both the shear rate and the temperature profile can lead to spatial variations in the final crystal morphology, and consequently non-uniform material properties - an undesirable consequence in manufacturing. Thus, establishing rules that forecast flow effects based on the processing conditions and material properties, as presented in this paper, are of extensive practical use.

There are a number of modelling efforts focused on describing flow-enhanced crystallization under typical processing conditions ${ }^{4,5}$, which incorporate the formation of multiple crystal phases ${ }^{6}$. There also exist numerous fundamental studies investigating flow-enhanced crystallization under controlled conditions; see $\operatorname{Ref}^{7}$ for a recent comprehensive review of the literature. Many of these fundamental studies investigate how crystals develop during an isothermal 'simple' flow, for example see Refs. ${ }^{8-12}$. The crystallization kinetics are monitored either directly, by measuring the number and size of spherulites that appear over time, or indirectly by measuring the rheological or optical response of the material during crystallization. Usually the melt is cooled to a crystallization temperature, a shear or extensional flow is applied, and crystallization is measured during the flow. Since the overshoot in the polymer stress is typically fast compared to crystallization kinetics, the flow reaches steady state during the observation window. Thus, the measured nucleation rate is constant in time and increases with increasing strain rate.

On the other hand, in typical polymer processing methods such as polymer extrusion ${ }^{13,14}$, injection molding ${ }^{15,16}$, film blowing ${ }^{17,18}$, fibre production ${ }^{19}$, and extrusion-based three-dimensional (3D) printing ${ }^{20}$, processing temperatures are usually above the melting point of the polymer, and crystallization follows in the absence of flow under non-isothermal conditions. Thus, the nucleation rate is typically non-constant, depending on both the decaying temperature 
and the flow-induced polymer deformation, which will begin to relax at the cessation of flow. Fundamental crystallization studies have employed similar protocols. A step shear may be applied at the crystallization temperature ${ }^{21,22}$, so that crystallization proceeds isothermally during polymer relaxation. Or more comparable to processing conditions, a step shear flow may be applied above the melting temperature, and the sample subsequently quenched to a crystallization temperature ${ }^{23,24}$. This 'short-term-shear' protocol is widely credited to Janeschitz-Kreigl ${ }^{2}$. Novel flow apparatus has been developed to impose transient stresses similar to those encountered in polymer processing and provide in-situ monitoring of microstructure development after cessation of flow ${ }^{25,26}$. For small strains, the shear time is typically less than the polymer crystallization (induction) time, so that the crystallization and the flow dynamics occur separately. Thus, this protocol presents an simpler modelling challenge in that the degree of crystallinity does not need to be coupled to the rheology.

In this paper, we present a continuum-level model for non-isothermal polymer crystallization following the cessation of a 'complex' flow applied for some period at a fixed temperature above the melting point. Here we focus on pipe flow; we are interested in how the prescribed quench protocol couples with the flow geometry, and how this interplay can affect the observed crystallization kinetics. The model is applicable to linear mono-disperse polymer melts, and may be extended to any flow geometry and subsequent cooling protocol. Here we focus only on the development of spherulite structures during crystallization, as described by the classical Schneider rate equations. Whilst the full numerical model is required for quantitative predictions of the induction time and spherulite-size distribution, we present a fundamental rule that can be used to determine the conditions or material properties required to guarantee, or eliminate, flow-enhanced spherulites a priori.

For instance, both the fundamental rule and full numerical model may be applied to injection moulding where flow is stopped when the cavity is filled and the cooling process commences, as well as in extrusion-based 3D printing of filaments where cooling is driven by natural convection in the surrounding air in the absence of flow; in particular, we summarise the results of Refs. ${ }^{27,28}$ in the discussion section. Further to previous work, in this paper we show how a semi-analytical solution to the heat equation enables the fundamental rule to be related simply to the processing conditions, without the need for the full numerical model. Thus, this rule provides a practical tool for determining the importance of flow-enhanced crystallization for a range of processes involving pipe flow. 


\section{A FUNDAMENTAL RULE}

Whilst it is well known that polymer nucleation is extremely sensitive to polymer deformation $^{7}$, the appropriate order parameter that governs enhanced nucleation remains an open question ${ }^{29}$. Here we use an empiricism derived from kinetic-Monte-Carlo simulations ${ }^{30}$, which suggests that nucleation is enhanced by the polymer stretch, denoted $\Lambda$. That is

$$
\dot{N}(T, \Lambda)=\dot{N}_{q}(T)\left(1+\eta\left(\Lambda^{4}-1\right)\right),
$$

where $\dot{N}_{q}$ is the quiescent nucleation rate and depends on the temperature profile, $T$. The fitting parameter $\eta$ governs the total number of extra nuclei created due to flow. Due to the generality of the model it is straight-forward to adapt the order parameter in Eq.1 based on emerging theories.

Since polymer stretch is governed by the polymer Rouse time $\tau_{R}$, stretching behaviour during a flow is characterized by the Rouse Weissenberg number:

$$
W i_{R}=\dot{\gamma} \tau_{R}
$$

for some strain rate $\dot{\gamma}$. That is, polymer molecules become stretched and may affect the nucleation rate via Eq.1 provided $W i_{R} \geq 1$. In the protocol of interest here, flow occurs at a fixed processing temperature temperature, denoted $T_{p}$. Thus, we define the Weissenberg number for $\tau_{R} \equiv \tau_{R}\left(T_{p}\right)$.

Whilst the Weissenberg number reveals if the flow is sufficiently strong the stretch the polymers, it does not tell us if this deformation persists at the onset of nucleation i.e. once the material has cooled to the melting temperature, denoted $T_{m}$, after the cessation of flow. Thus, we propose that an additional dimensionless parameter is required to forecast the importance of flow on the following crystallization kinetics: the ratio of the time taken for the material to cool to the melting temperature, which we denote $\tau_{m}$, to the stretch relaxation time, $\tau_{R}$. Since $\tau_{m}$ may be considered as the characteristic time scale for the process, this additional parameter is in fact an inverse Deborah number:

$$
D e^{-1}=\frac{\tau_{m}}{\tau_{R}},
$$

and polymer stretch persists at the onset of nucleation only if $D e^{-1}<1$.

In typical processes, the temperature decays from the processing temperature, $T_{p}$, to some quench temperature, denoted $T_{q}$. Thus, we define $D e^{-1}$ at $T_{a v}=\left(T_{p}+T_{q}\right) / 2$, i.e. 


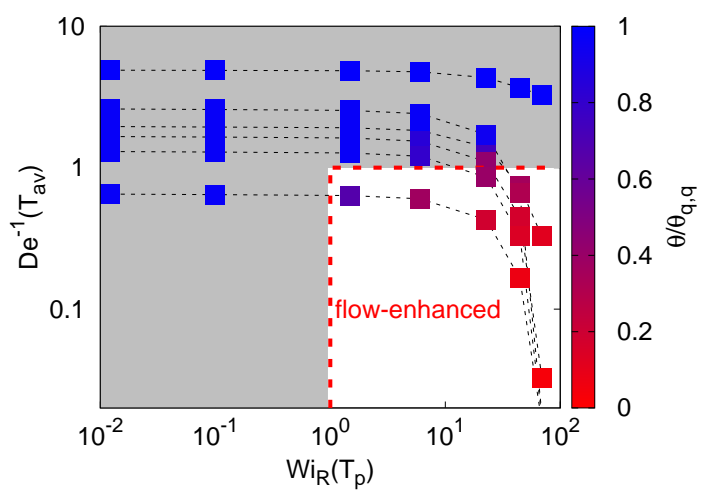

FIG. 1. A phase diagram comparing the Weissenberg number, $W i_{R}$ (Eq.2), to the time available for flow-enhanced crystallization, $D e^{-1}$ (Eq.3). Flow-enhanced crystallization only occurs if $W i_{R}>1$ and $D e^{-1}<1$. The colour scale corresponds to the induction time (time to reach full space filling) normalised by the quiescent isothermal induction time (Eq.A15), as predicted by the full numerical model i.e. $\theta / \theta_{q, q}<1$ corresponds to flow-enhanced crystallization. Each black line represents variations along a pipe radius for various 'typical' processing conditions.

by taking $\tau_{R} \equiv \tau_{R}\left(T_{a v}\right)$. Whilst establishing the Rouse time, $\tau_{R}$, and its temperature dependence is well-known for linear polymers ${ }^{31}$, the time to reach the melting point, $\tau_{m}$, is more difficult to calculate. Thus, further to previous work, in Sec.IV we show how $\tau_{m}$ can be determined semi-analytically for pipe flow, and expressed in terms of the processing conditions and material properties. Consequently, we can derive the following fundamental rule for determining the importance of flow on proceeding polymer crystallization, based on known material properties and processing conditions. That is, flow-enhanced crystallization will only be observed if

$$
W i_{R}\left(T_{p}\right) \geq 1 \quad \text { AND } \quad D e^{-1}\left(T_{a v}\right)<1 .
$$

For example, Fig.1 illustrates a typical phase diagram arising from crystallization of a linear polymer within a quenched pipe following flow, as predicted by our full numerical model to be discussed in the next section. Both $W i_{R}$ and $D e^{-1}$ vary across the pipe, with $W i_{R}$ increasing and $D e^{-1}$ decreasing as you near the pipe wall. Each black line represents the variation from the centre of the pipe to the pipe wall under various conditions (discussed in further detail in Sec.V). The color scale corresponds to the crystallization induction time, denoted $\theta$, defined to be the time taken for spherulites to approach full space filling. The induction time is non-dimesionalized relative to the equivalent crystallization time under 
quiescent (no stretch) isothermal conditions, denoted $\theta_{q, q}$ (see Eq.A15 for details of how to calculate this time scale analytically). That is, $\theta / \theta_{q, q}<1$ corresponds to flow-enhanced crystallization, meaning a higher nucleation density and therefore smaller spherulites. Fig.1 clearly shows where spatial inhomogeneities in the spherulite size will arise across the pipe radius due to flow effects, and where flow-enhanced crystallization is suppressed despite $W i_{R}>1$.

\section{A NUMERICAL MODEL}

\section{A. Overview}

The numerical model presented here is constructed to replicate flow applied to a monodisperse polymer melt, followed by cooling in the absence of flow. In this way flow and crystallization are decoupled, and the model constitutes the following two-stage dynamics:

1. Stage 1: Flow is applied to the material at some processing temperature, $T_{p}$, which is chosen to be above the melting point, $T_{m}$. The polymer molecules stretch and orient in response to the strain rate, $\dot{\gamma}$, and eventually reach steady state according the some constitutive equation (the Rolie-Poly model ${ }^{32}$ is used in this work). The temperature remains uniform in both space and time.

2. Stage 2: The flow is switched off $(\dot{\gamma}=0)$ and the material cools from $T_{p}$ to the quench temperature, $T_{q}$, according to a prescribed temperature protocol (defined by a cooling time scale, $\left.\tau_{c}\right) ; T_{q}$ may be above or below the glass transition temperature, $T_{g}$, of the material. During this time any deformation of the polymer molecules will relax. At $T=T_{m}$, the polymers will nucleate and spherulites will grow according to the Schneider rate equations ${ }^{33}$. In the case $T_{q}>T_{g}$, crystallization will proceed to full space filling, as captured by the Avrami equation ${ }^{34}$.

The governing equations of the model have been described previously ${ }^{27}$, and are therefore detailed in Appendix A. The model contains only a single fitting parameter, $\eta$ (found in Eq.1); all other parameters can be derived from materials characterization. A list of measurements required to obtain the model parameters is given in Table I. (Quantitative

values for typical polymers used in extrusion-based printing are given in Appendix B.) The parameters $T_{p}, T_{q}, \tau_{c}$ and $\dot{\gamma}$ are determined by the process conditions. 
TABLE I. Measurements required for obtaining the model parameters. The values for poly-lactic acid (PLA) and polycaprolactone (PCL) used in extrusion-based printing are listed in Appendix B.

\begin{tabular}{|c|c|c|c|}
\hline Measured Quantity & Notation & Model Parameters & Notation \\
\hline \multicolumn{4}{|l|}{ Material Properties } \\
\hline Elastic Moduli & $G^{\prime}, G^{\prime \prime}$ & $\begin{array}{c}\text { Tube Model Parameters } \\
\text { (entanglement } M_{w} \text { and time, } \\
\text { plateau modulus) }\end{array}$ & $M_{e}, \tau_{e}^{0}, G_{e}$ \\
\hline WLF parameters & $C_{1}, C_{2}, T_{r e f}$ & Shift Factor & $a(T)(\mathrm{Eq} \cdot \mathrm{A} 8)$ \\
\hline Molecular Weight & $M_{w}$ & Entanglement Number & $Z=M_{w} / M_{e}$ \\
\hline & & Relaxation Times & $\tau_{d}, \tau_{R}$ (Eq.A7\&A9) \\
\hline \multicolumn{4}{|l|}{ Thermal Diffusivity } \\
\hline Thermal Conductivity & $k$ & Thermal Diffusivity & $\alpha=k / \rho C_{p}$ \\
\hline Specific Heat Capacity & $C_{p}$ & & \\
\hline Density & $\rho$ & & \\
\hline \multicolumn{4}{|c|}{ Crystallization Parameters } \\
\hline Crystal growth rate & $G(T)$ & Crystal growth parameters & $G_{\max }, b_{g}$ (Eq.A13) \\
\hline Quiescent nucleation rate & $\dot{N}_{q}(T)$ & Nucleation parameters & $n_{0}, b_{n}$ (Eq.A14) \\
\hline
\end{tabular}

Since flow through channels or pipes is ubiquitous in industrial processes, in the following section we discuss the appropriate boundary conditions required to model steady axisymmetric pipe flow (although the model can readily extended to any flow geometry and unsteady flows), followed by a quench in the temperature profile. We consider the case where $T_{q}>T_{g}$; the case $T_{q}<T_{g}$ is considered in Sec.VI. During Stage 2, we focus solely on the development of spherulite structures. Furthermore, it should be noted that the model neglects the affect of pressure. In particular, the high pressure induced in capillary flow is known to affect the crystallization temperature in some polymers more than others ${ }^{35}$. The shear rate/flow speed will also affect the pressure required to drive the flow and consequently may affect the crystallization kinetics. This effect will be considered in future work. 

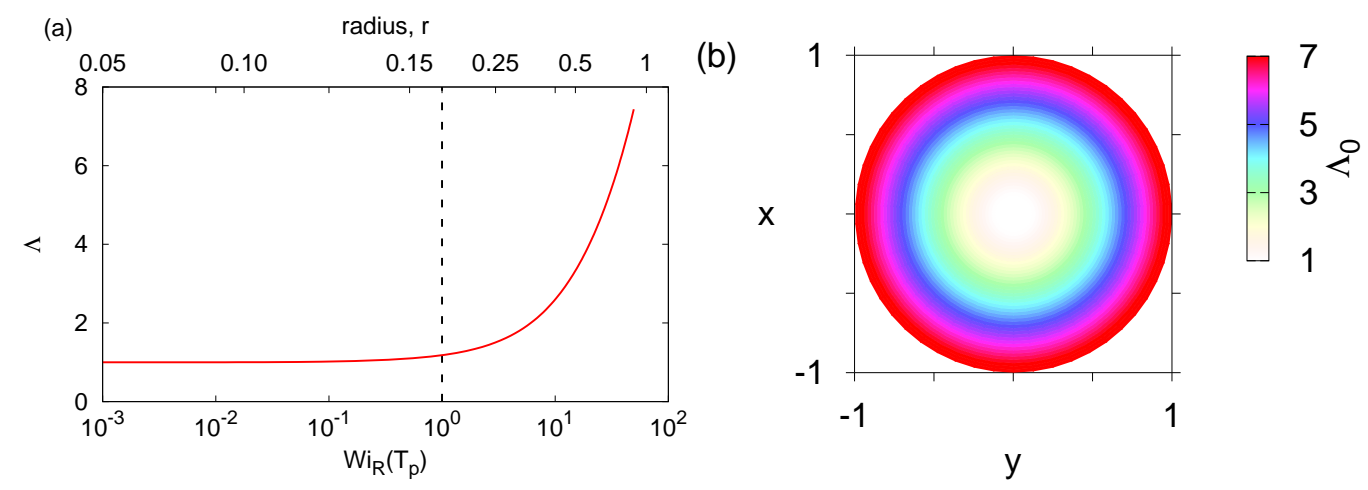

FIG. 2. (a) Polymer stretch $\Lambda$ as a function of the Weissenberg number, $W i_{R}$. The dimensionless radius $r$ at which this Weissenberg number is achieved during steady pipe flow for some pressure gradient $\partial p / \partial z$ is labelled, and the corresponding pipe cross-section is shown in (b).

\section{B. Stage 1: Steady Axisymmetric Pipe Flow}

For steady axisymmetric pipe flow we use a polar coordinate system $\mathbf{r}=(r, \theta, z)$. The velocity field is given by $\mathbf{u}=(0,0, w(r))$, where $w(r)$ is to be determined. We apply a no-slip boundary condition at the nozzle wall $r=R$ such that

$$
\begin{aligned}
& w(r=R)=0 \\
& w(r=0) \text { is bounded, }
\end{aligned}
$$

and solve the conservation of momentum equation

$$
\frac{\partial p}{\partial z}=\frac{1}{r} \frac{\partial}{\partial r}\left(r \sigma_{r z}\right)
$$

for constant pressure gradient, $\partial p / \partial z$, and shear stress, $\sigma_{r z}$. The pressure gradient determines the average speed of the material, as it flows through the nozzle. During the flow we assume a constant processing temperature $T=T_{p},\left(T_{p}>T_{m}\right)$, which enters the constitutive model for the shear stress (see Appendix A).

Fig.2(a) demonstrates the steady-state polymer stretch, $\Lambda_{0}$, achieved as a function of the Rouse Weissenberg number (Eq.2). Polymers only become stretched once $W i_{R}\left(T_{p}\right)$ exceeds unity. Note that finite-extensibility has been neglected here, but may be added to the constitutive equation (Eq.A6). The corresponding radius at which this Weissenberg number is achieved during a typical pipe flow is also labelled. Fig.2(b) shows the corresponding cross-sectional stretch profile within a pipe of radius $R$. 
This steady state solution of Stage 1 provides an initial condition for the Stage 2 cooling, relaxation and crystallization dynamics, as discussed in Appendix A. In the following section, we discuss the boundary conditions required to solve the evolving temperature profile across the pipe radius. (Note that the relaxation and crystallization dynamics are detailed in Appendix A and do not require boundary conditions.)

\section{Stage 2: Temperature Protocols}

We assume axisymmetric cooling of the pipe, so that the heat equation can be written as

$$
\frac{\partial T}{\partial t}=\alpha \frac{1}{r} \frac{\partial}{\partial r}\left(r \frac{\partial T}{\partial r}\right)+\frac{H_{f}}{C_{p}} \dot{\xi}, \quad T(\mathbf{r}, t=0)=T_{p} .
$$

The internal cooling time scale of the material is defined by the ratio of thermal diffusivity, $\alpha$, to the area of the pipe, such that

$$
\tau_{\alpha}=\frac{R^{2}}{\alpha}
$$

The second term of the right-hand side of Eq.8 defines latent heat effects; $H_{f}$ denotes the latent heat of crystallization, $C_{p}$ is the specific heat capacity, and $\dot{\xi}$ is the crystallization rate.

We prescribe the boundary at $r=R$ to cool exponentially in time from the processing temperature $T_{p}$ to the quench temperature $T_{q}$ such that

$$
T(r=R)=\left(T_{p}-T_{q}\right) \exp \left(-t / \tau_{c}\right)+T_{q}
$$

Here $\tau_{c}$ is some specified external cooling time. The limit of short cooling times $\tau_{c} \rightarrow 0$ provides the Dirichlet condition

$$
T(r=R)=T_{q}
$$

which we expect to be similar to cooling at a solid wall interface i.e. when the material remains within the pipe during the quench. On the other hand, long cooling times $\tau_{c}>$ $\tau_{\alpha}$ reflect conditions at a polymer-air interface, i.e. the conditions on a polymer filament extruded through a pipe into air (e.g. see Ref. ${ }^{27}$ ). Note that at $r=0$ the solution must be bounded.

Further details of how the cooling protocol couples with the polymer relaxation and crystallization are given in Appendix A. 


\section{RESUlTS I: PREDICTING THE TIME TO THE MELTING POINT}

In order for the fundamental rule described in Sec.II to be of practical use, we require an analytical solution for the time taken to reach the melting point, namely $\tau_{m}$, which appears in $D e^{-1}$ (Eq.3). Thus, we require a time-dependent solution to the heat equation (Eq.8) with $\dot{\xi}=0$ (as there is no crystallization whilst $T>T_{m}$ ). Since the exact solution to heat equation contains a Bessel function, which must be inverted numerically, here we seek a semi-analytic solution that employs a polynomial approximation to the Bessel's function that is chosen empirically.

First, we non-dimensionalise the heat equation via

$$
\Theta=\frac{T-T_{q}}{T_{p}-T_{q}} \quad ; \quad \tilde{t}=\frac{t}{\tau_{\alpha}} \quad ; \quad \tilde{r}=\frac{r}{R}
$$

where ${ }^{\sim}$ denotes a dimensionless variable. With $\dot{\xi}=0$, this yields

$$
\frac{\partial \Theta}{\partial \tilde{t}}=\frac{1}{\tilde{r}} \frac{\partial}{\partial \tilde{r}}\left(\tilde{r} \frac{\partial \Theta}{\partial \tilde{r}}\right), \quad \Theta(\tilde{\mathbf{r}}, \tilde{t}=0)=1
$$

together with the boundary condition

$$
\Theta(\tilde{r}=1)=\exp \left(-\frac{\tau_{\alpha}}{\tau_{c}} \tilde{t}\right) .
$$

First, we consider the boundary condition $\tau_{c} \rightarrow 0$, i.e.

$$
\Theta(\tilde{r}=1)=0
$$

so that the time to reach time melting temperature at $\tilde{r}=1$ is $\tilde{\tau}_{m}=0$. At the centre of the pipe $\tilde{r}=0$, the cooling of the material is determined solely by its thermal diffusivity. In light of the imposed boundary condition, we assume that temperature decay is approximately exponential at the centre, so that

$$
\Theta(\tilde{r}=0) \approx \exp (-\tilde{t})
$$

Thus, the time taken to reach the melting temperature at the centre of the pipe is given by

$$
\tilde{\tau}_{m}(\tilde{r}=0)=-\ln \Theta_{m}
$$

where $\Theta_{m}=\left(T_{m}-T_{q}\right) /\left(T_{p}-T_{q}\right)$. 

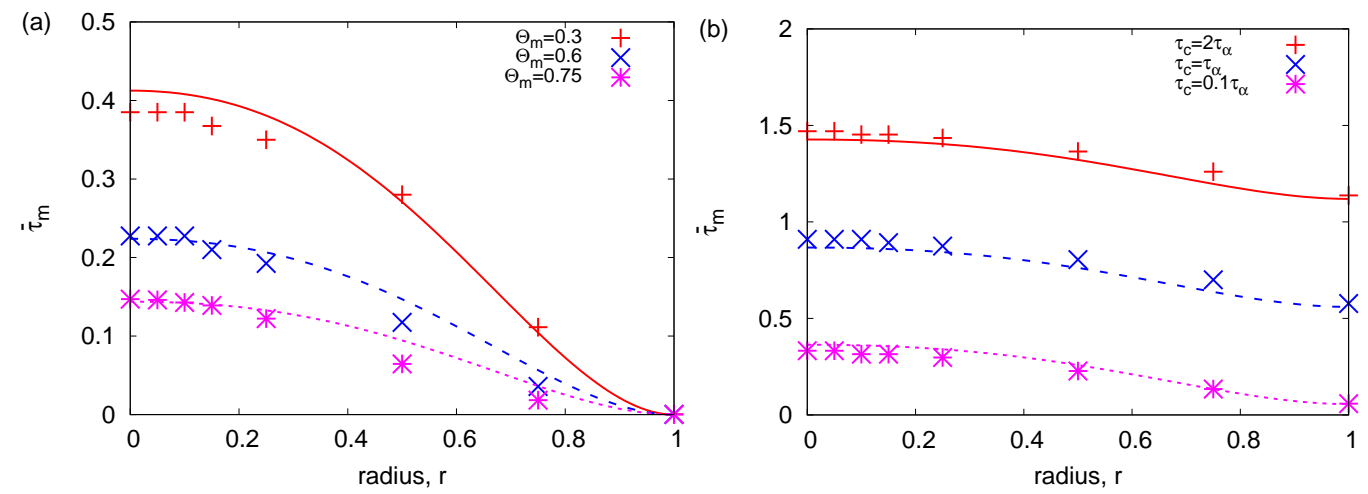

FIG. 3. Time taken to reach the melting temperature, $\tilde{\tau}_{m}$, as a function of the dimensionless nozzle radius, $r$, compared to semi-analytic solution given by Eq.20 for different values of the cooling time scale and $\Theta_{m}$ (a) $\tau_{c}=0$ with $\Theta_{m}=0.3,0.6,0.75$, (b) $\Theta_{m}=0.6$ with $\tau_{c}=2 \tau_{\alpha}, \tau_{\alpha}, 0.1 \tau_{\alpha}$.

We then extend this solution for any $\tilde{r} \in(0,1]$ by assuming the spatial solution takes the form of a polynomial, $f(r)$, such that

$$
\tilde{\tau}_{m}(r)=-A \ln \Theta_{m} f(\tilde{r})
$$

where $A$ is some constant. We find empirically, that $f(\tilde{r})$ takes the form

$$
f(\tilde{r})=\left(1-\tilde{r}^{2}\right)\left(1-\tilde{r}^{3}\right)
$$

and $A \approx 0.5$, as shown in Fig.3.

Thus, in general, the time to reach the melting temperature can be written as

$$
\tilde{\tau}_{m}(\tilde{r})=-\ln \Theta_{m}\left(A f(\tilde{r})+\frac{\tau_{c}}{\tau_{\alpha}}\right)
$$

Fig.3 shows how numerical solutions for the time to reach the melting temperature in a pipe compares to the semi-analytic solution (Eq.20) for different values of $\Theta_{m}$ and cooling ratio $\tau_{c} / \tau_{\alpha}$, typical for polymer processing (in particular extrusion-based 3D printing ${ }^{27}$ ).

Thus, Eq.20 can be used to calculate $D e^{-1}$ (Eq.3) and therefore employed to construct phase diagrams for various processing conditions and material properties, similar to that shown earlier (Fig.1). Next, we consider how the spatial dependence of $\tilde{\tau}_{m}$ can affect the development of spherulites during cooling, for which the full numerical model is required. 


\section{RESULTS II: FLOW-ENHANCED CRYSTALLIZATION}

In this section we employ the full numerical model to demonstrate how the induction time and spherulite size can vary across the pipe radius due to the interplay between the Rouse Weissenberg number, $W i_{R}$ (Eq.2), and the time available for flow-enhanced crystallization as determined by $D e^{-1}$ (Eq.3). In particular, the initial condition on the polymer stretch due to flow through the pipe is shown in Fig.2(b), and we apply an exponential decay of the temperature profile at the boundary according to Eq.10, and vary the magnitude of the cooling time scale, $\tau_{c}$. For example, Fig.4 shows the model results for $\tau_{c}=0.1 \tau_{\alpha}$. The time variable is scaled with the quiescent isothermal induction time at the quench temperature, denoted $\theta_{q, q}$ (as given by Eq. A15), so that $\theta / \theta_{q, q}<1$ corresponds to flowenhanced crystallization.

Fig.4(a) shows how the temperature across the pipe decays; the time to reach the melting temperature $\tau_{m}$ increases towards the centre of the pipe. Fig.4(b) shows the corresponding stretch relaxation curves; the molecules at $r=1$ begin with a greater degree of stretch and relax much slower due to the lower temperature.

In Fig.4(c) we can see how the onset is nucleation is delayed until $T<T_{m}$. Since there is no stretch at $r=0$, the nucleation rate is only affected by the decrease in temperature. On the other hand, for larger $r$ the nucleation rate is enhanced due to the increase in polymer stretch. Enhanced nucleation ceases once the stretch has relaxed.

Fig.4(d) shows how the degree of space filling evolves over time for each $r$. Crystallization is accelerated nearer to the nozzle wall due to the increase in nucleation density induced by the increased polymer stretch. At $r=0$ we recover quiescent (no stretch kinetics), and, since the temperature is uniform at the onset of crystal growth, the induction time at $r=0$ does not differ much from the induction time evaluated at constant temperature, $\theta_{q, q}$ (Eq.A15). This accelerated crystallization as a function of $r$ affects the cross-sectional crystal morphology.

In particular, Fig.5 shows how the spherulite size distribution changes for different values of the cooling time scale, $\tau_{c}$, with fixed initial stretch profile. (Fig.5(b) corresponds to the dynamics shown in Fig.4).A boundary layer of smaller slow-enhanced spherulites can be seen in some cases, and the thickness of this boundary varies significantly with the cooling protocol. It is clear to see how the assumption of axisymmetry of both the flow and 

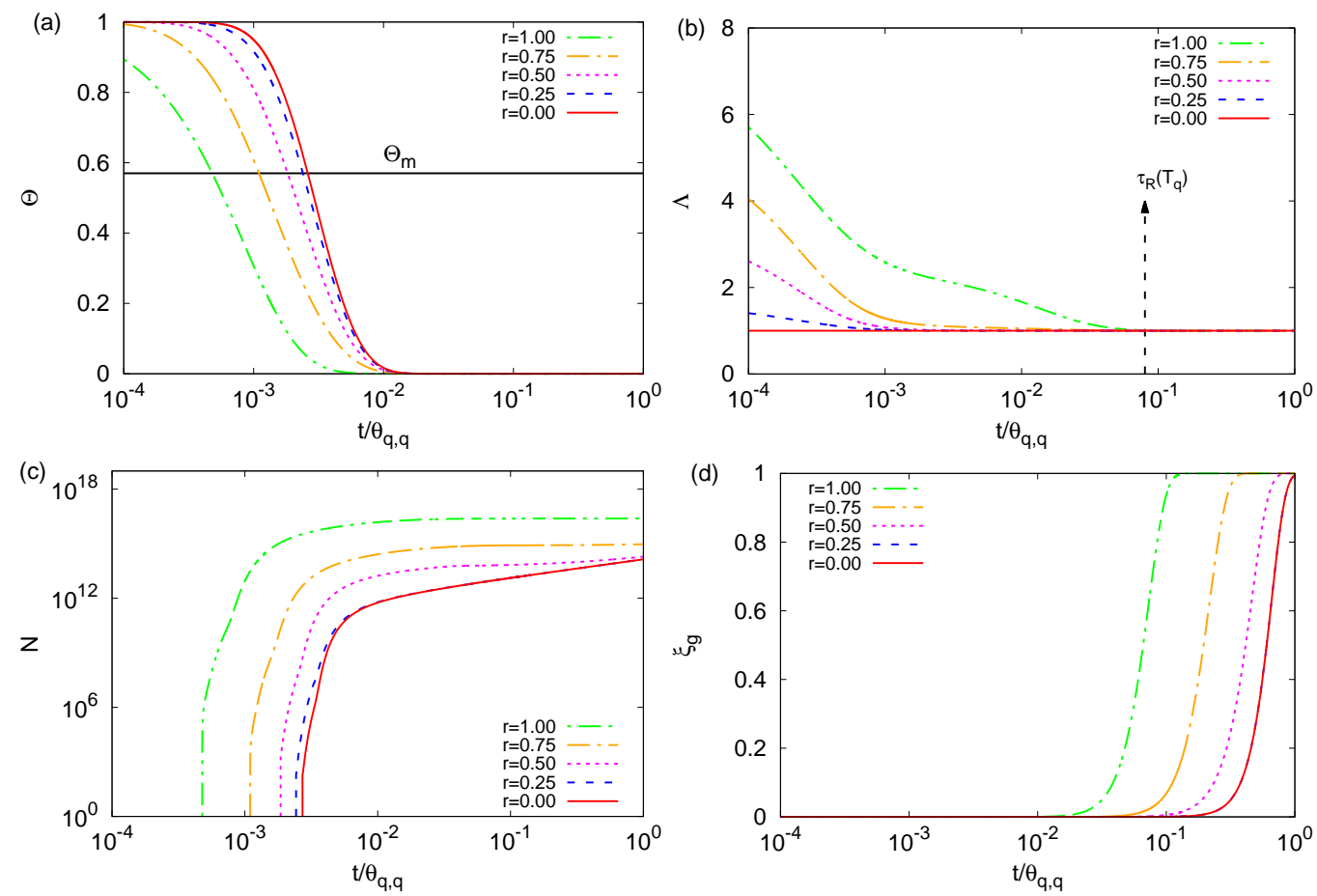

FIG. 4. (a) Dimensionless temperature evolution, $\Theta$, (b) relaxation of the polymer stretch, $\Lambda$, (c) number of nuclei created, $N$, and (d) degree of space filling, $\xi_{g}$, as a function of time. Time is non-dimensionalised by the quiescent isothermal induction time $\theta_{q, q}$ (Eq.A15). The polymer nucleates once $\Theta<\Theta_{m}$, and crystallization is enhanced near to the nozzle walls $(r \rightarrow 1)$ due to residual polymer stretch at the onset of nucleation. In this example, we set $\tau_{c}=0.1 \tau_{\alpha}$.

cooling profiles leads to symmetry in the spherulite distribution. The framework presented here may be readily adapted to explore how non-axisymmetric cooling profiles affect flowenhanced crystallization i.e. by adapting Eq.10. Moreover, flow-enhanced crystallization can be completely eliminated by increasing the cooling time so that $\tau_{m}>\tau_{R}$ i.e. the stretch is fully relaxes at the onset of nucleation. This demonstrates that the time to reach the melting temperature as a function of space, namely $\tau_{m} \equiv \tau_{m}(r)$, is key to determining the cross-sectional crystal morphology.

Thus, in addition to the Rouse Weissenberg number (Eq.2), we have demonstrated the importance of defining an additional parameter - the inverse Deborah number $D e^{-1}$ (Eq.3) - to determine the importance of flow on subsequent crystallization kinetics. The behaviour shown in Fig.5 is mapped to the phase diagram given in Fig.6, again with the color scale corresponding to the reduced induction time $\theta / \theta_{q, q}$. 
(a)
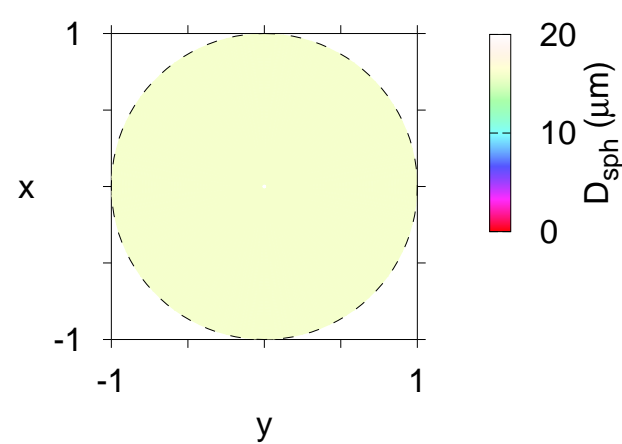

(c)



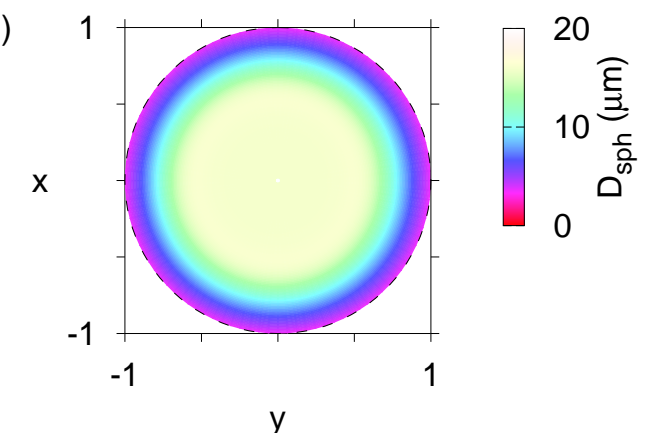

20

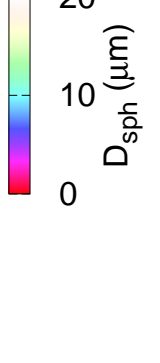

FIG. 5. Distribution of spherulites diameters $D_{s p h}$ across the pipe radius for different cooling times (a) $\tau_{c}=\tau_{\alpha}$, (b) $\tau_{c}=0.1 \tau_{\alpha}$, and (c) $\tau_{c}=0.01 \tau_{\alpha}$

Importantly, despite the approximations made in Sec.IV in order to derive a solution for the time to reach the melting point, $t_{m}$, our fundamental rule is in quantitative agreement with the full numerical model. As discussed earlier, flow-enhanced crystallisation only occurs when

$$
W i_{R}\left(r, T_{p}\right) \geq 1 \quad \text { AND } \quad D e^{-1}\left(r, T_{a v}\right)<1
$$

and each black line shows the variation in crystallization kinetics across the pipe radius. (Note that the earlier phase diagram (Fig.1) also contains this data, together with results for the case $\tau_{c}=0$ coupled with a range of thermal diffusivity time scales, $\tau_{\alpha}$.) This fundamental rule has also been validated against experimental measurements of crystallization during extrusion-based 3D printing ${ }^{27,28}$, although $\tau_{m}$ was previously calculated numerically; these results are summarised in Sec.VIC.

\section{DISCUSSION}

In this section, we first discuss how the presence of flow-enhanced spherulites can be controlled by the material rheology (in addition to the processing parameters). Second, we 


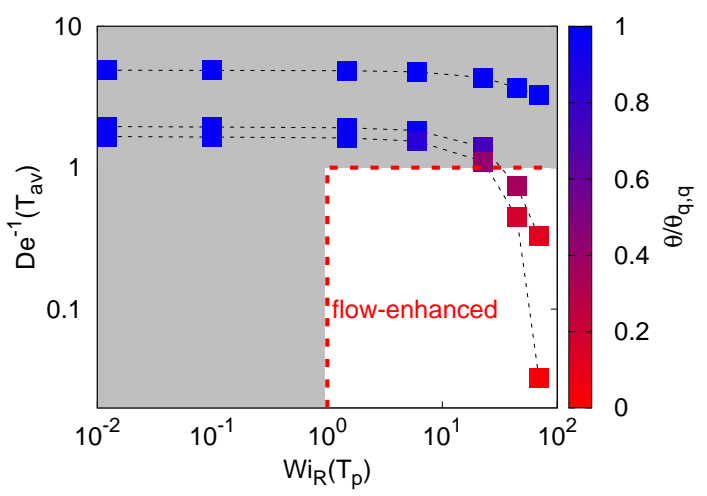

FIG. 6. Phase diagram corresponding to results shown in Fig.5, comparing the Weissenberg number, $W i_{R}$ (Eq.2), to the time available for flow-enhanced crystallization, $D e^{-1}$ (Eq.3). Flowenhanced crystallization only occurs if $W i_{R}>1$ and $D e^{-1}<1$. The colour scale corresponds to the induction time (time to reach full space filling) normalised by the quiescent isothermal induction time (Eq.A15), as predicted by the full numerical model. Each black line represents variations along a pipe radius for the conditions shown in Fig.5

demonstrate how fast cooling such that $T_{q}<T_{g}$ may inhibit crystallisation during processing, but how variations in the nucleation density may be 'templated' by the flow; consequently, flow-enhanced spherulites can be revealed by post-processing annealing and also predicted by the fundamental rule presented here. Finally, we discuss applications of the model to extrusion-based 3D printing.

\section{A. Controlling Flow-Enhanced Crystallization}

We have seen that controlling the onset of flow-enhanced crystallization is determined by

$$
W i_{R}=\dot{\gamma} \tau_{R}>1 \quad \text { and } \quad D e^{-1}=\frac{\tau_{m}}{\tau_{R}}<1
$$

Thus, we can vary the following processing parameters to control where in the pipe flowenhanced crystallization occurs:

- the shear rate, $\dot{\gamma}$, via varying the pressure gradient $\partial p / \partial z$ or altering the pipe radius, $R$, and/or

- the external cooling time scale, $\tau_{c}$. 

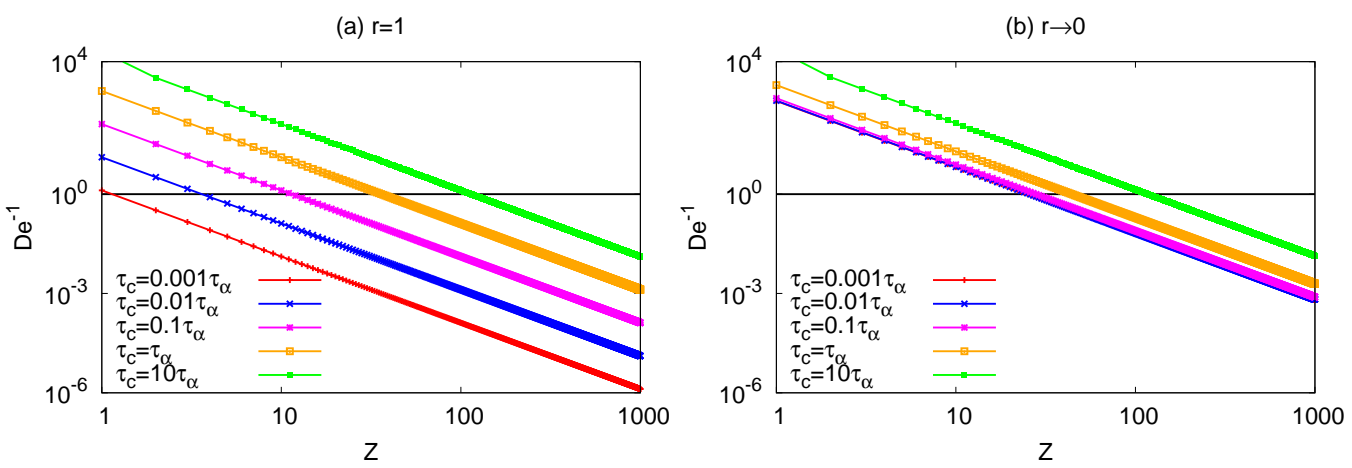

FIG. 7. Inverse Deborah number $D e^{-1}$ (Eq.3)

at (a) nozzle wall, $r=1$, and (b) the centre of the pipe, $r=0$, as a function of the molecular weight of the material (or equivalently the number of entanglements, $Z$ (Eq.23), for various cooling times, $\tau_{c}$.

On the other hand, if the process conditions are considered fixed, then we can vary the molecular weight, $M_{w}$, of the material to ensure that flow-enhanced spherulites are achieved. Since linear polymer melts consist of a number of entangled segments of molecular weight $M_{e}$, increasing the molecular weight increases the total number of entanglement per polymer chain

$$
Z=\frac{M_{w}}{M_{e}}
$$

and consequently increases the Rouse time of the material (see Eq.A9). For reference, the results in Sec.V correspond to $Z=20$. Thus, in the following, we demonstrate how $D e^{-1}$ varies with $Z$.

Since $D e^{-1}$ depends on both nozzle size $R$ and cooling time $\tau_{c}$, in this example we show results for representative nozzle radius, $R=200 \mu \mathrm{m}$, and a number of cooling times $\tau_{c}$, relative to the thermal diffusivity time $\tau_{\alpha}$. In Figs.7(a) and (b), we show results at the nozzle wall $r=1$, where cooling is governed by the external boundary condition, i.e.

$$
\tau_{m}(r=1)=-\ln \left(\frac{T_{m}-T_{q}}{T_{p}-T_{q}}\right) \tau_{c},
$$

and at the centre $r=0$, where cooling is governed by internal thermal diffusion, i.e.

$$
\tau_{m}(r=0)=-\ln \left(\frac{T_{m}-T_{q}}{T_{p}-T_{q}}\right)\left(A \tau_{\alpha}+\tau_{c}\right) .
$$

Suppose hypothetically that at the nozzle wall the shear rate is always large enough to induce stretch so that $W i_{R}>1, \forall Z$. Since the time to reach the melting temperature is 
determined by the boundary condition (Eq.24), we can ensure $D e^{-1}<1$ and therefore flowenhanced crystallization for any cooling time $\tau_{c}$, by increasing the entanglement number $Z$, as shown in Fig.7(a). In fact, flow-enhanced crystallization will occur for any $Z$ provided $\tau_{c} \rightarrow 0$

On the other hand, it is much more difficult to induce flow-enhanced crystallization in the center of pipe. In steady pipe flow, for example, the polymers always remain at equilibrium at $r=0$ and will therefore not affect the crystallization kinetics at the pipe center. In the unsteady state, on the other hand, contractions in the pipe will induce an extensional flow, and therefore polymer stretch, at $r=0$. For sufficiently strong flows, the Rouse Weissenberg number will thus exceed unity in the centre of the pipe. Nevertheless, even if $W i_{R}>1$ is achieved, it remains difficult to achieved flow-enhanced spherulites at $r=0$ due to the following reason.

As shown in Fig.7(b), regardless of decreasing the external cooling rate such that $\tau_{c} \rightarrow 0$, it is impossible to ensure $D e^{-1}<1$ for $Z<25$ for the pipe geometry chosen here. In fact, since the time to reach the melting temperature at the center of the pipe is also governed by $\tau_{\alpha}, D e^{-1}$ becomes independent of $\tau_{c}$. Consequently, ensuring $\tau_{m}<\tau_{R}$ for small $Z$ means decreasing the pipe radius (assuming thermal diffusivity is a fixed material parameter). This is an important consideration when designing experiments to probe flowinduced crystallization under typical processing conditions.

\section{B. Templating Crystallinity}

In this section, we consider the effect of a quench temperature $T_{q}<T_{g}$. In this case crystallization is arrested once $T \leq T_{g}$ and there is little time for the spherulites to grow. However, nucleation will occur during $T_{m} \geq T \geq T_{g}$ and will be affected by any residual polymer stretch persisting from the flow. As shown in Fig.8(a), there is a clear boundary of flow-enhanced nuclei near to the surface, which have not had sufficient time to grow into spherulites.

Both flow-induced and quiescent nuclei are 'templated' into the material and can be grown into spherulites via post-processing annealing. Usually the annealing temperature is chosen so that there is little nucleation, and the annealing time is chosen to be sufficiently long that full space filling is achieved. As shown in Fig.8(b), the annealing process reveals 
(a) Before Annealing:
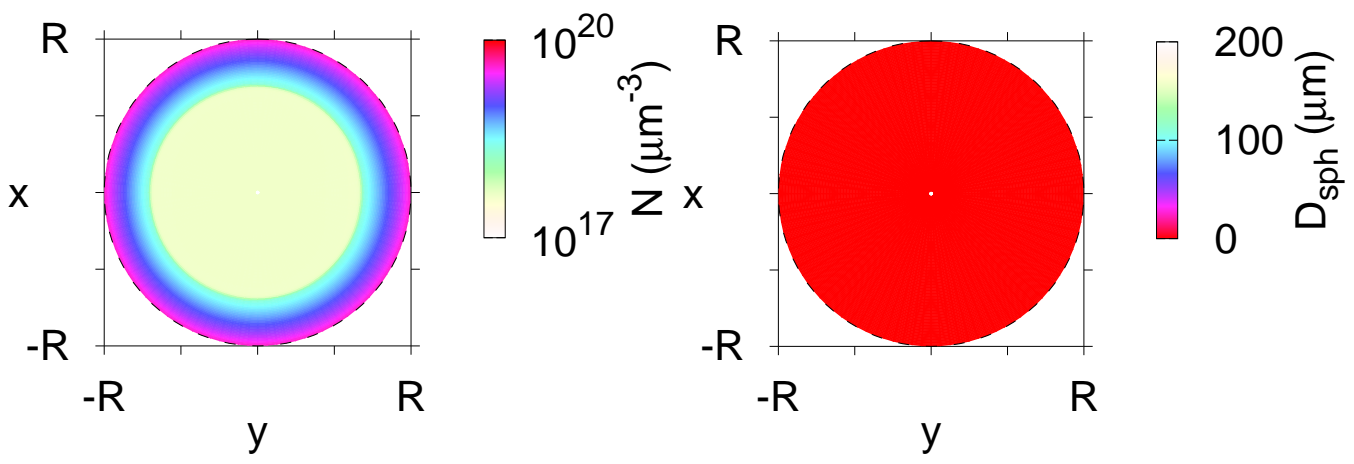

(b) After Annealing:
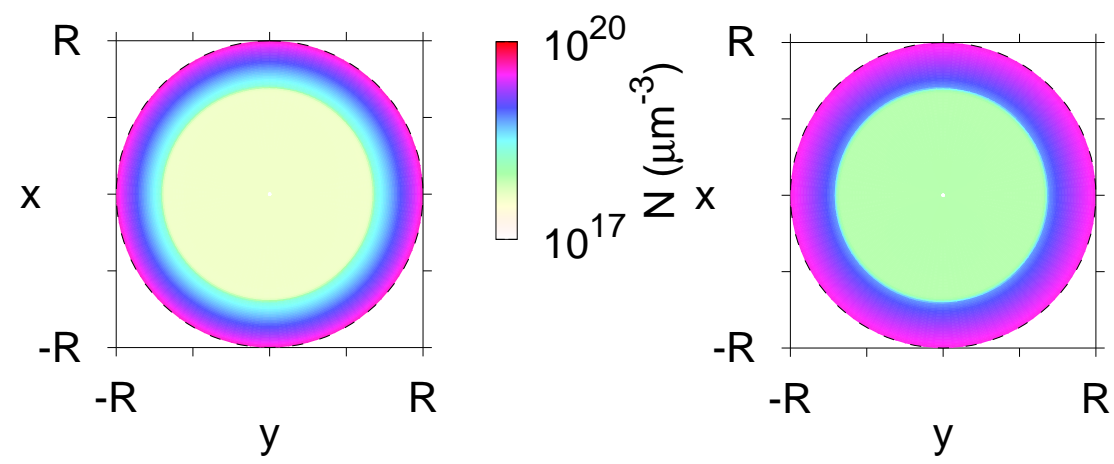

FIG. 8. The number of nuclei, $N$, and the spherulite diameter, $D_{s p h}$, (a) before annealing and (b) after an annealing process which allows spherulites to reach full space filling. Flow-enhanced nuclei are 'templated' by the flow and only revealed after annealing.

these smaller flow-induced spherulites near to $r=1$, which have a similar distribution to those shown in Fig.5. Moreover, the fundamental rule presented in this paper can also be employed to ensure or eliminate this templated crystallinity phenomenon.

\section{Applications in Extrusion-Based Printing}

Extrusion-based 3D printing, also know as fused filament fabrication (FFF), remains the cheapest additive manufacturing technique, and has the desired ability to process these highperformance thermoplastics for high-end applications. The FFF process involves filamentby-filament extrusion of molten plastic, which rapidly cools and solidifies in the absence of flow. Advanced application of FFF is limited by the strength of printed parts; in particular weld regions at filament-filament interfaces are notoriously weak. Understanding the ultimate crystal morphology is crucial to determining the part strength, and the model described 


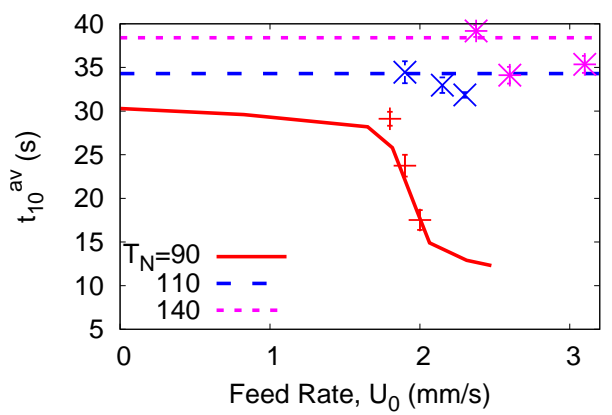

FIG. 9. Time to reach $10 \%$ crystallization for PCL as a function of print speed for three print temperatures. At higher temperatures, the polymer stretch becomes fully relaxed before the temperature reaches the melting point so there is no flow-enhanced crystallization. Reprinted from Additive Manufacturing, 24, McIlroy \& Graham, 'Modelling flow-enhanced crystallixation during fused filament fabrication of semi-crystalline polymer melts', 323-340, Copyright 2018, with permission from Elsevier.

a)

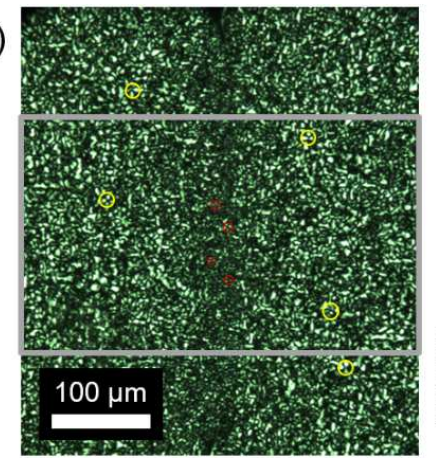

b)

c)
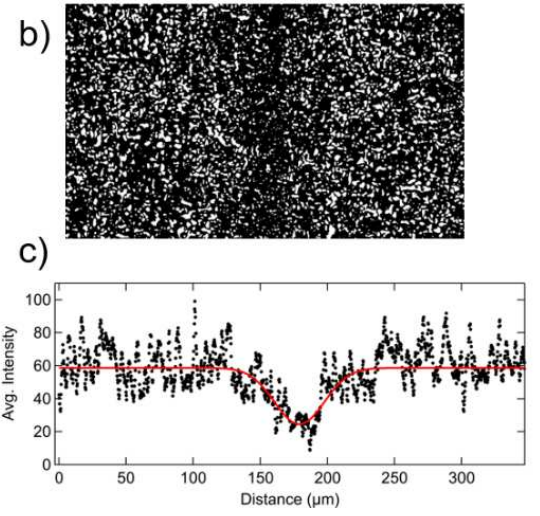

$\mathrm{T}_{\mathrm{N}}=200^{\circ} \mathrm{C}$

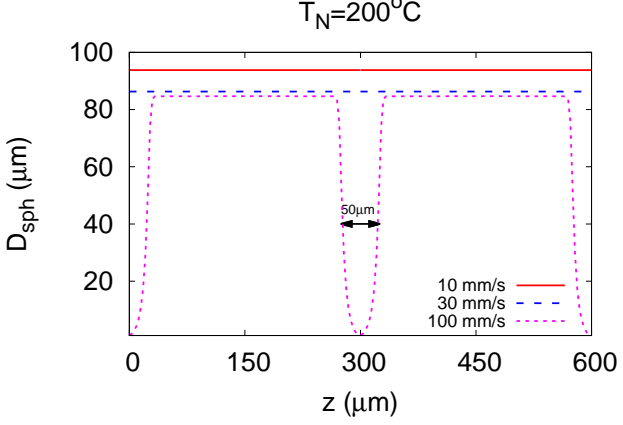

FIG. 10. (a) Annealed cross-section of PLA part printed at $200^{\circ} \mathrm{C}$ and $10 \mathrm{~mm} / \mathrm{s}$. b) The thresholded, binarized image of the selected region. c) The pixel intensity as a function of distance. The points indicate the average pixel intensity and the line indicates the Gaussian fit to the intensity profile. (d) Corresponding model predictions of the spherulite diameter, $D_{s p h}$, as a function of distance, z.Reprinted with permission from Ref. ${ }^{28}$. Copyright 2019 American Chemical Society. 
above has been successfully applied to FFF for a number of different materials ${ }^{27,28}$.

In particular, we have shown how the polymer stretch induced by FFF can accelerate crystallization times under certain processing conditions ${ }^{27}$. As shown in Fig.9, the predicted induction times are in quantitative agreement with in-situ Raman spectroscopy measurements of polycaprolactone (PCL) for a range of different processing conditions (print temperature and print speed). The model highlights important features not captured by a single measurement of the induction time. In particular, the crystal morphology varies cross-sectionally, with smaller spherulites forming in an outer skin layer, whilst the bulk of filament is governed by slower quiescent kinetics. The thickness of this flow-enhanced boundary layer is determined by the thermal diffusivity of the polymer, and may facilitate improving the strength at filament-filament interfaces.

The model has also been successfully applied to FFF-printing of poly-lactic acid (PLA) ${ }^{28}$. PLA behaves quite differently from PCL. One reason for this is that PLA has a much higher glass transition temperature - above room temperature - that will arrest crystallization during printing. This is not the case for PCL, which has a low glass transition temperature allowing crystallization to proceed to full space filling. In fact, our flow-enhanced crystallization model shows that whilst flow-induced nuclei are 'templated' into the deposited filament near to the free surface during printing, there is insufficient time for spherulites to grow. To explore the effect of this templated nucleation density, we model a typical post-printing thermal annealing process to initiate spherulite growth and allow full space filling to be achieved. As shown in Fig.10, for the first time a molecularly-aware modelling approach for predicting the crystal morphology within an annealed printed filament is compared to optical microscopy images for a range of printing conditions, revealing smaller flow-enhanced spherulites in the weld regions between filaments.

\section{CONCLUSION}

Understanding variations in crystallinity and spherulite size is crucial to determining the properties and uniformity of parts produced by polymer processing. In this paper we discuss typical processing conditions that involve flowing a material under 'hot' conditions, followed by quenching and flow-enhanced crystallization in the absence of flow. We present the results given by our general continuum-level model, which is able to quantify inhomogeneous 
variations spherulite size due to the effects of both flow and temperature gradients. The model contains only a single fitting parameter, can be readily adapted to different polymer materials, flow geometries and cooling protocols, and has successfully been applied to 3Dprinting flows.

Although the full numerical model is required to make quantitative predictions of the crystallization process, we propose that only a simple fundamental rule is required to predict the presence of flow-enhanced spherulites a priori. Since the Rouse Weissenberg number only determines if the flow is sufficiently strong to stretch polymers, an additional parameter,

$D e^{-1}$, which considers the time available for flow-enhanced crystallization, is also required to determine the importance of flow prior to polymer crystallization. More precisely, $D e^{-1}$ determines if there is residual stretch at the onset of nucleation that will accelerate crystallization. Further to previous works, we have shown that the process or cooling time scale contained in $D e^{-1}$ can be determined semi-analytically in pipe flow. Future work will consider if similar analysis extends to various flow geometries and cooling protocols, including non-axisymmetric cooling.

This rule can be used to forecast the possibility of inhomogeneities in the degree of crystallinity and the spherulite size, without the need for a full numerical model. In fact, flow-enhanced structures can be guaranteed or eliminated by varying $D e^{-1}$, through material rheology, cooling protocol or flow geometry. Furthermore, under the conditions where fast cooling inhibits crystal growth during processing, this fundamental rule can be applied to determine if flow-enhanced structures will be revealed due to post-processing thermal annealing.

\section{ACKNOWLEDGMENTS}

The author would like to thank Richard Graham for many useful discussions about flowenhanced polymer crystallization. This research was funded by the Royal Commission for the Exhibition of 1851. 


\section{Appendix A: Governing Equations}

The model presented here consists of equations for a steady flow at constant temperature, as well as the simultaneous cooling, relaxation, and crystallization of the polymers in the absence of flow.

\section{Flow Equation}

To calculate the flow field $\mathbf{u}$, we solve conservation of mass

$$
\nabla \cdot \mathbf{u}=0
$$

coupled with conservation of momentum

$$
\nabla \cdot \boldsymbol{\sigma}=0
$$

for stress tensor $\boldsymbol{\sigma}$. Here the time derivative is set to zero for steady-state behaviour.

In a polymer melt, the stress field consists of an isotropic pressure, along with solvent and polymer contributions to the stress such that

$$
\boldsymbol{\sigma}=-p \mathbf{I}+\mu_{s}\left(\mathbf{K}+\mathbf{K}^{T}\right)+G_{e}(\mathbf{A}-\mathbf{I}) .
$$

Here $\mathbf{K}=\nabla \mathbf{u}$ is the velocity gradient tensor. The solvent viscosity and elastic modulus are denoted $\mu_{s}$ and $G_{e}$, respectively, and the polymer configuration tensor $\mathbf{A}$ is defined by

$$
\mathbf{A}=\frac{<\mathbf{R R}>}{2 R_{g}},
$$

where $\mathbf{R}$ is the end-to-end vector of the polymer chain and $R_{g}$ is the chain radius of gyration. In this way, the polymer stretch is given by

$$
\Lambda=\sqrt{\frac{\operatorname{tr} \mathbf{A}}{3}}
$$

and the off-diagonal elements of $\mathbf{A}$ determine the polymer orientation.

To couple the flow field to the polymer stress, we employ the Rolie-Poly model ${ }^{32}$ as it is proven to quantitatively predict both reptation and stretch relaxation behaviour of monodisperse polymer chains within a melt. The steady-state Rolie-Poly equation is defined by

$$
\mathbf{K} \cdot \mathbf{A}+\mathbf{A} \cdot \mathbf{K}^{T}-\frac{1}{\tau_{d}(T)}(\mathbf{A}-\mathbf{I})-\frac{2}{\tau_{R}(T)}\left(1-\frac{1}{\Lambda}\right)\left(\mathbf{A}+\frac{\beta}{\Lambda}(\mathbf{A}-\mathbf{I})\right)=0
$$


where $\tau_{d}$ and $\tau_{R}$ denoted the reptation time and stretch relaxation (Rouse) times, respectively. The convective constraint release parameter $\beta$ is set to unity in this work to avoid shear banding instabilities in the flow region.

Finally the flow is coupled to the temperature field $T$ through the polymer relaxation times. The reptation time $\tau_{d}$ governs the orientation of the tube, and is given by

$$
\tau_{d}(T)=\tau_{e}^{0} Z^{2} a(T)
$$

where the time-temperature-superposition factor $a(T)$ can be described by either the Arrenius law or the Williams-Landel-Ferry (WLF) equation. In this work we use a simple WLF equation:

$$
a(T)=\exp \left(-\frac{C_{1}\left(T-T_{0}\right)}{C_{2}+T-T_{0}}\right) .
$$

However, it should be noted that there is evidence that the WLF equation does not apply in the vicinity of the glass transition temperature ${ }^{36}$.

The Rouse time $\tau_{R}$ governs the relaxation of the tube stretch, and is given by

$$
\tau_{R}(T)=3 \tau_{e}^{0} Z^{3}\left(1-\frac{3.38}{\sqrt{Z}}+\frac{4.17}{Z}-\frac{1.55}{\sqrt{Z}^{3}}\right) a(T) .
$$

In Eqs.A7 \& A9, $\tau_{e}^{0}$ is the Rouse time of one entanglement segment at $T_{0}$. Both $M_{e}$ and $\tau_{e}^{0}$ can be extracted by fitting the linear rheology data $\left(G^{\prime}, G^{\prime \prime}\right)$ to the Likhtman \& McLeish model $^{37}$, and is executed using Reptate software ${ }^{38}$.

\section{Crystallization Kinetics}

Since we are interested in crystallization following flow, velocity gradients are set to zero (i.e. $\mathbf{K}=\mathbf{0}$ ), and any polymer deformation relaxes via

$$
\frac{d \mathbf{A}}{d t}=\frac{1}{\tau_{d}(T)}(\mathbf{A}-\mathbf{I})-\frac{2}{\tau_{R}(T)}\left(1-\frac{1}{\Lambda}\right)\left(\mathbf{A}+\frac{\beta}{\Lambda}(\mathbf{A}-\mathbf{I})\right) .
$$

Crystallisation of the polymer molecules is well described by the Schneider Rate equations:

$$
\begin{aligned}
& \dot{\phi}_{3}=8 \pi \dot{N}(T, \Lambda), \quad\left(\phi_{3}=8 \pi N\right) \\
& \dot{\phi}_{2}=G(T) \phi_{3}, \quad\left(\phi_{2}=8 \pi R_{t o t}\right) \\
& \dot{\phi}_{1}=G(T) \phi_{2}, \quad\left(\phi_{1}=S_{t o t}\right) \\
& \dot{\phi}_{0}=G(T) \phi_{1}, \quad\left(\phi_{0}=V_{t o t}\right),
\end{aligned}
$$


where $N$ denotes the total number of nuclei, and $R_{t o t}, S_{t o t}$ and $V_{\text {tot }}$ denote the total radii, surface area and volume fraction of spherulites that grow over time. The crystal growth and nucleation rates are denoted $G$ and $\dot{N}$, respectively. To correct for space filling, the Avrami equation is employed

$$
\phi_{0}=-\ln \left(1-\xi_{g}\right)
$$

where $\xi_{g}$ denotes the degree of space filling. Note that due to the decoupling of the flow and crystallization kinetics in this model, it is not necessary for the degree of space filling to feed back into the polymer rheology. In other works, this coupling is acheived by either adapting the plateau modulus ${ }^{39}$, or the relaxation times ${ }^{5}$.

The crystal growth rate, $G$, depends only on temperature, $T$, and is usually described by the Lauritzman-Hoffman theory

$$
G(T)=\frac{G_{0}}{a(T)} \exp \left(\frac{-b_{g}}{T\left(T_{m}-T\right)}\right)
$$

for constants $G_{0}$ and $b_{g}$, and melting temperature $T_{m}$. The temperature-shift factor $a(T)$ (Eq.A8) ensures crystallization is arrested near to the glass transition temperature $T_{g}$.

In quiescent (no deformation) conditions, the nucleation rate is described similarly:

$$
\dot{N}_{q}(T)=\frac{n_{0}}{a(T)} \exp \left(\frac{b_{n}}{T-T_{m}}\right) .
$$

Here $n_{0}$ and $b_{n}$ are constants. The enhanced nucleation rate due to polymer stretch is then given by Eq.1. Usually Eqs. A13 \& A14 are fit to experimental data.

We define the induction time, $\theta$, to be the time taken for the system to approach full spacing filling i.e. $\xi_{g} \rightarrow 1$. For a constant nucleation rate (i.e. $\Lambda=1$ and uniform temperature), the induction time can be calculated analytically by integrating the Schneider rate equations. That is the quiescent (no stretch) induction time at the quench temperature is given by

$$
\theta_{q, q}=\lim _{\xi_{g} \rightarrow 1}\left(-\frac{3}{\pi} \frac{\ln \left(1-\xi_{g}\right)}{G\left(T_{q}\right)^{3} \dot{N}_{q}\left(T_{q}\right)}\right)^{0.25}
$$

This provides an appropriate time scale with which to non-dimensionalise time. Flowenhanced crystallization is thus indicated by $\theta / \theta_{q, q}<1$, as described earlier (Fig.1). 


\section{Temperature Equation}

The temperature field is calculated by solving the heat equation:

$$
\frac{\partial T}{\partial t}=\alpha \nabla^{2} T+\frac{H_{f}}{C_{p}} \dot{\xi}
$$

where $\alpha$ is the thermal diffusivity of the material, $H_{f}$ is the latent heat of crystallization, $C_{p}$ is the specific heat capacity, and $\dot{\xi}$ is the crystallization rate.

\section{Appendix B: Printing Material Parameters}

Poly-lactic acid (PLA) and polycaprolactone (PCL) are used in extrusion-based 3D printing applications. Here we list the model parameters, corresponding to Table I, for these two printing materials. Further details can be found in Refs. ${ }^{27,28}$.

TABLE II. Model parameters for a typical semi-crystalline printing material poly-lactic acid (PLA), obtained either directly from the literature or by fitting to experimental data available in the literature. See Ref. $^{28}$ for further details.

\begin{tabular}{l|c|c|c}
\hline \hline PLA Property & Notation & Value & Units \\
\hline Molecular Weight & $M_{w}$ & 103.3 & $\mathrm{~kg} \mathrm{~mol}-1$ \\
Entanglement Molecular Weight & $M_{e}$ & 4.0 & $\mathrm{k} \mathrm{mol}^{-1}$ \\
Plateau Modulus & $G_{e}$ & $8.7 \times 10^{5}$ & $\mathrm{~Pa}$ \\
Entanglement Time (at $\left.T_{0}\right)$ & $\tau_{e}^{0}$ & $6.7 \times 10^{-6}$ & $\mathrm{~s}$ \\
\hline Reference Temperature & $T_{0}$ & 180 & ${ }^{\circ} \mathrm{C}$ \\
WLF parameter & $C_{1}$ & 7.4 & ${ }^{\circ} \mathrm{C}^{-1}$ \\
WLF parameter & $C_{2}$ & 175.2 & ${ }^{\circ} \mathrm{C}$ \\
\hline Crystal growth rate parameters & $G_{m a x}$ & $4.9 \times 10^{-8}$ & $\mathrm{~m}^{\mathrm{s}}$ \\
\hline Nucleation parameters & $b_{g}$ & 23.1 & ${ }^{\circ} \mathrm{C}^{-2}$ \\
\hline Thermal Diffusivity & $n_{0}$ & $2.1 \times 10^{16}$ & $\mathrm{~m}^{-3} \mathrm{~s}^{-1}$ \\
\hline
\end{tabular}


TABLE III. Model parameters for semi-crystalline printing material polycaprolactone (PCL), often used in medical applications, obtained either directly from the literature or by fitting to experimental data available in the literature. See Ref. ${ }^{27}$ for further details.

\begin{tabular}{l|c|c|c}
\hline \hline PCL Property & Notation & Value & Units \\
\hline Molecular Weight & $M_{w}$ & 96.7 & $\mathrm{~kg} \mathrm{~mol}^{-1}$ \\
Entanglement Molecular Weight & $M_{e}$ & 4.8 & $\mathrm{k} \mathrm{mol}^{-1}$ \\
Plateau Modulus & $G_{e}$ & $9.2 \times 10^{5}$ & $\mathrm{~Pa}$ \\
Entanglement Time (at $\left.T_{0}\right)$ & $\tau_{e}^{0}$ & $1.9 \times 10^{-5}$ & $\mathrm{~s}$ \\
\hline Reference Temperature & $T_{0}$ & 60 & ${ }^{\circ} \mathrm{C}$ \\
WLF parameter & $C_{1}$ & 6 & ${ }^{\circ} \mathrm{C}^{-1}$ \\
WLF parameter & $C_{2}$ & 123 & ${ }^{\circ} \mathrm{C}$ \\
\hline Crystal growth rate parameters & $G_{m a x}$ & $4.78 \times 10^{-4}$ & ${ }^{\mathrm{m} / \mathrm{s}}$ \\
\hline Nucleation parameters & $b_{g}$ & 0.0043 & ${ }^{\circ} \mathrm{C}^{-2}$ \\
\hline Thermal Diffusivity & $n_{0}$ & $1.8 \times 10^{16}$ & $\mathrm{~m}^{-3} \mathrm{~s}^{-1}$ \\
\hline
\end{tabular}

\section{REFERENCES}

${ }^{1}$ E. Piorkowska and G. C. Rutledge, Handbook of polymer crystallization (John Wiley \& Sons, 2013).

${ }^{2}$ H. Janeschitz-Kriegl and E. Ratajski, "Some fundamental aspects of the kinetics of flowinduced crystallization of polymers," Colloid and Polymer Science 288, 1525-1537 (2010).

${ }^{3}$ J. Way, J. Atkinson, and J. Nutting, "The effect of spherulite size on the fracture morphology of polypropylene," Journal of Materials Science 9, 293-299 (1974).

${ }^{4}$ P. C. Roozemond, M. van Drongelen, Z. Ma, M. A. Hulsen, and G. W. Peters, "Modeling flow-induced crystallization in isotactic polypropylene at high shear rates," Journal of rheology 59, 613-642 (2015).

${ }^{5}$ F. J. M. F. Custdio, R. J. A. Steenbakkers, P. D. Anderson, G. W. M. Peters, and H. E. H. Meijer, "Model development and validation of crystallization behavior in injection molding prototype flows," Macromolecular Theory and Simulations 18, 469-494 (2009). 
${ }^{6}$ P. C. Roozemond, T. B. van Erp, and G. W. Peters, "Flow-induced crystallization of isotactic polypropylene: Modeling formation of multiple crystal phases and morphologies," Polymer 89, 69-80 (2016).

${ }^{7}$ R. S. Graham, "Understanding flow-induced crystallization in polymers: A perspective on the role of molecular simulations," Journal of Rheology 63, 203-214 (2019).

${ }^{8}$ R. Pantani, I. Coccorullo, V. Volpe, and G. Titomanlio, "Shear-induced nucleation and growth in isotactic polypropylene," Macromolecules 43, 9030-9038 (2010).

${ }^{9}$ C. Hadinata, C. Gabriel, M. Ruellman, and H. Laun, "Comparison of shear-induced crystallization behavior of pb-1 samples with different molecular weight distribution," Journal of Rheology 49, 327-349 (2005).

${ }^{10}$ S. Coppola, L. Balzano, E. Gioffredi, P. L. Maffettone, and N. Grizzuti, "Effects of the degree of undercooling on flow induced crystallization in polymer melts," Polymer 45, 3249-3256 (2004).

${ }^{11}$ E. E. B. White, H. H. Winter, and J. P. Rothstein, "Extensional-flow-induced crystallization of isotactic polypropylene," Rheologica acta 51, 303-314 (2012).

${ }^{12}$ R. H. Somani, B. S. Hsiao, A. Nogales, S. Srinivas, A. H. Tsou, I. Sics, F. J. Balta-Calleja, and T. A. Ezquerra, "Structure development during shear flow-induced crystallization of i-pp: in-situ small-angle x-ray scattering study," Macromolecules 33, 9385-9394 (2000).

${ }^{13}$ R. Toukhtarian, S. G. Hatzikiriakos, H. Atsbha, and B. Boulet, "Modeling polymer extrusion with varying die gap using arbitrary lagrangian eulerian (ale) method," Physics of Fluids 30, 093103 (2018).

${ }^{14}$ V. K. Konaganti, M. Derakhshandeh, M. Ebrahimi, E. Mitsoulis, and S. G. Hatzikiriakos, "Non-isothermal extrudate swell," Physics of Fluids 28, 123101 (2016).

${ }^{15}$ R. Mollaabbasi, E. Behzadfar, and S. Taghavi, "A simplified semi-analytical model for the filling and cooling process in plastic molding," Physics of Fluids 31, 063105 (2019).

${ }^{16}$ P. Poungthong, A. J. Giacomin, C. Saengow, C. Kolitawong, H.-C. Liao, and S.-C. Tseng, "Nonlinear core deflection in injection molding," Physics of Fluids 30, 053102 (2018).

${ }^{17}$ A. K. Doufas and A. J. McHugh, "Simulation of film blowing including flow-induced crystallization," Journal of Rheology 45, 1085-1104 (2001).

${ }^{18} \mathrm{~T}$. Barborik and M. Zatloukal, "Effect of heat transfer coefficient, draw ratio, and die exit temperature on the production of flat polypropylene membranes," Physics of Fluids 31, 053101 (2019). 
${ }^{19}$ J. Drabek and M. Zatloukal, "Meltblown technology for production of polymeric microfibers/nanofibers: A review," Physics of Fluids 31, 091301 (2019).

${ }^{20}$ E. Bertevas, J. Férec, B. C. Khoo, G. Ausias, and N. Phan-Thien, "Smoothed particle hydrodynamics (sph) modeling of fiber orientation in a 3d printing process," Physics of Fluids 30, 103103 (2018).

${ }^{21}$ J. Baert, P. V. Puyvelde, and F. Langouche, "Flow-induced crystallization of pb-1: from the low shear rate region up to processing rates," Macromolecules 39, 9215-9222 (2006).

${ }^{22}$ S. Acierno, S. Coppola, and N. Grizzuti, "Effects of molecular weight distribution on the flow-enhanced crystallization of poly (1-butene)," Journal of Rheology 52, 551-566 (2008).

${ }^{23}$ F. G. Hamad, R. H. Colby, and S. T. Milner, "Onset of flow-induced crystallization kinetics of highly isotactic polypropylene," Macromolecules 48, 3725-3738 (2015).

${ }^{24}$ B. Nazari, H. Tran, B. Beauregard, M. Flynn-Hepford, D. Harrell, S. T. Milner, and R. H. Colby, "Two distinct morphologies for semicrystalline isotactic polypropylene crystallized after shear flow," Macromolecules 51, 4750-4761 (2018).

${ }^{25}$ G. Kumaraswamy, R. K. Verma, and J. A. Kornfield, "Novel flow apparatus for investigating shear-enhanced crystallization and structure development in semicrystalline polymers," Review of scientific instruments 70, 2097-2104 (1999).

${ }^{26}$ M. Seki, D. W. Thurman, J. P. Oberhauser, and J. A. Kornfield, "Shear-mediated crystallization of isotactic polypropylene: The role of long chain- long chain overlap," Macromolecules 35, 2583-2594 (2002).

${ }^{27}$ C. McIlroy and R. Graham, "Modelling flow-enhanced crystallisation during fused filament fabrication of semi-crystalline polymer melts," Additive Manufacturing 24, 323-340 (2018).

${ }^{28}$ J. E. Seppala, A. P. Kotula, and C. R. Snyder, "Polymer-based additive manufacturing: Recent developments. acs symposium series," (2019).

${ }^{29}$ D. A. Nicholson and G. C. Rutledge, "An assessment of models for flow-enhanced nucleation in an n-alkane melt by molecular simulation," Journal of Rheology 63, 465-475 (2019).

${ }^{30}$ R. S. Graham and P. D. Olmsted, "Coarse-grained simulations of flow-induced nucleation in semicrystalline polymers," Phys. Rev. Lett. 103, 115702 (2009).

${ }^{31}$ M. Doi and S. F. Edwards, The theory of polymer dynamics (Oxford University Press, Oxford, 1988). 
${ }^{32}$ A. E. Likhtman and R. S. Graham, "Simple constitutive equation for linear polymer melts derived from molecular theory: Rolie-Poly equation," Journal of Non-Newtonian Fluid Mechanics 114, 1-12 (2003).

${ }^{33}$ G. W. Peters, L. Balzano, and R. J. Steenbakkers, "Flow-induced crystallization," Handbook of polymer crystallization , 399-431 (2013).

${ }^{34}$ M. Avrami, "Kinetics of phase change. ii transformationtime relations for random distribution of nuclei," The Journal of Chemical Physics 8, 212-224 (1940).

${ }^{35} \mathrm{~K}$. Kishore and R. Vasanthakumari, "A comprehensive review of the effect of pressure on polymer crystallization," High Temperatures-High Pressures 16, 241-268 (1984).

${ }^{36}$ V. Jadhao and M. O. Robbins, "Probing large viscosities in glass-formers with nonequilibrium simulations," Proceedings of the National Academy of Sciences 114, 7952-7957 (2017).

${ }^{37}$ A. E. Likhtman and T. C. McLeish, "Quantitative theory for linear dynamics of linear entangled polymers," Macromolecules 35, 6332-6343 (2002).

${ }^{38}$ A. Likhtman, "Reptate," https://reptate.readthedocs.io/ (2019).

${ }^{39}$ J. Seo, H. Takahashi, B. Nazari, A. M. Rhoades, R. P. Schaake, and R. H. Colby, "Isothermal flow-induced crystallization of polyamide 66 melts," Macromolecules 51, 4269-4279 (2018). 